\title{
Fatores que influenciam o desmame precoce e a extensão do aleitamento materno
}

\section{The early weaning and extended breastfeeding influent factors}

\author{
Karina Camillo CARRASCOZA \\ Áderson Luiz COSTA JÚNIOR \\ Antônio Bento Alves de MORAES
}

\begin{abstract}
Resumo
O objetivo deste trabalho foi identificar fatores que influenciam a ocorrência do desmame precoce e do aleitamento prolongado. A metodologia envolveu a seleção de dois grupos de 40 mães, cujos filhos eram atendidos pelo Centro de Pesquisa e Atendimento Odontológico para Pacientes Especiais, Faculdade de Odontologia de Piracicaba, Universidade Estadual de Campinas. O grupo I era constituído de mães que desmamaram seus filhos antes do sexto mês de vida e o grupo II, de mães que estenderam a amamentação além do primeiro ano. As participantes responderam, primeiramente, a entrevistas individuais por meio de um questionário específico e, posteriormente, a um questionário socioeconômico. A entrevista foi gravada em fita de áudio para evitar a perda de informações verbais. Os resultados mostraram que as variáveis demográficas estão relacionadas ao sucesso ou insucesso da amamentação natural. Programas de saúde que analisam essas variáveis podem identificar mulheres com alto risco para a ocorrência do desmame precoce e proporcionar-lhes uma atenção mais sistematizada.
\end{abstract}

Palavras-chave: amamentação; comportamento de cuidado da criança; crianças; mãe.

\begin{abstract}
The aim of this study was to identify influent factors on the early weaning and extended breastfeeding occurrences. The sample was composed by two groups of 40 mothers each, whose children were patients of the preventive program offered at Centro de Pesquisa e Atendimento Odontológico para Pacientes Especiais, Faculdade de Odontologia de Piracicaba Universidade Estadual de Campinas. Group I was consisted by mothers who had weaned before their children were six months old, and group II was consisted by mothers who had extended the breastfeeding beyond their baby's first year. A specific questionnaire was applied during individual interviews, and mothers also answered a Socioeconomic Questionnaire. In order to avoid information loss, the interviews were recorded and then, transcripted. Results points outs demographic aspects as variables of breastfeeding success. Health programs, that analyze these variables, may identify high-riskearly weaning mothers, and offer them a systematic attention, considering their individual needs.

Key words: breastfeeding; child care; child; mother.

$\boldsymbol{V} \boldsymbol{\nabla v}$

1 Doutoranda, Programa de Pós-Graduação em Saúde da Criança e do Adolescente, Faculdade de Ciências Médicas, Universidade Estadual de Campinas. Rua Tessalia Vieira de Camargo, 126, Cidade Universidade, 13083-887, Campinas, SP, Brasil. Correspondência para/Correspondence to: K.C. CARRASCOZA. E-mail: <karinacarras@ig.com.br>.

2 Instituto de Psicologia, Universidade de Brasília. Brasília, DF, Brasil.

3 Faculdade de Odontologia de Piracicaba, Universidade Estadual de Campinas. Piracicaba, SP, Brasil.

O trabalho foi financiado pelo CNPq.
\end{abstract}


O aleitamento materno constitui um dos pilares fundamentais para a promoção da saúde das crianças em todo o mundo, oferecendo vantagens não só para o bebê, como também para a mãe. A amamentação natural é um aliado na redução dos índices de mortalidade infantil, diminui a probabilidade de processos alérgicos e gastrintestinais nos primeiros meses de vida do bebê, proporciona melhores indicadores de desenvolvimento cognitivo e psicomotor, favorece o adequado desenvolvimento de estruturas da face, entre outros benefícios (Nascimento \& Issler, 2003). Para a mãe, reduz a probabilidade de ocorrência de câncer de mama, proporciona maior espaçamento entre os partos e uma involução uterina mais rápida, com conseqüente diminuição do sangramento pós-parto (Giugliane \& Victora, 2000).

As superioridades do leite humano, como fonte de alimento e de proteção contra doenças, e do ato de amamentar, como fonte de desenvolvimento afetivo, levaram os pesquisadores da área da saúde a recomendarem a amamentação natural exclusiva por seis meses de vida do bebê (Araújo, Fiaco, Pimenel \& Schmitz, 2004; Rezende, 2004; Updegrove, 2004). Mas, infelizmente, ainda tem se observado um alto índice de desmame precoce (anterior ao sexto mês de vida) em diversas populações (Dennis, 2002; Neighbors, Gillespie, Schwartz \& Foxman, 2003).

Observa-se que a ausência de amamentação ou sua interrupção precoce e a introdução de outros tipos de alimentos na dieta da criança têm sido freqüentes, com conseqüências potencialmente danosas à saúde do bebê, tais como a exposição precoce a agentes infecciosos, contato com proteínas estranhas e prejuízos ao processo de digestão (Pedroso, Puccini, Silva, Silva \& Alves, 2004).

A partir da década de 80, foram propostas diversas estratégias e levadas a efeito várias campanhas para aumentar a prevalência da amamentação no Brasil. Dados de pesquisas nacionais apontam para um incremento nos índices de aleitamento materno nas duas últimas décadas (Camilo, Carvalho, Oliveira \& Moura, 2004). No entanto, esse incremento inclui ampla variabilidade, conforme a região geográfica e as características sociodemográficas da população, o que sugere a necessidade de um maior conhecimento sobre

434 os padrões de amamentação de diferentes grupos populacionais. Informações mais sistemáticas poderiam permitir uma melhor avaliação dos serviços desenvolvidos e subsidiar mudanças e ajustes nas práticas de promoção e incentivo à amamentação natural (McKeever, Stevens, Miller, McDonell, Gibbins, Guerriere, Dunn \& Coyte, 2002).

Leite, Silva e Scochi (2004), por exemplo, chamam a atenção para a importância dos cursos de aconselhamento em amamentação, idealizados pelo Fundo das Nações Unidas para a Infância (UNICEF), em parceria com a Organização Mundial de Saúde (OMS), com o objetivo de valorizar a mulher como agente da amamentação, compreender e facilitar a aplicação de habilidades de comunicação não-verbal entre mãe e bebê e, ainda, prolongar o período de amamentação exclusiva. Para as autoras, é fundamental que os profissionais de saúde estejam atentos aos sinais não verbais da mulher, pois esses retratam suas emoções. Tais sinais podem ser indicadores das dificuldades que a mulher enfrenta, das interpretações que ela faz acerca de elementos interacionais em seu contexto e que, muitas vezes, são indicativos do curso que ela poderá imprimir ao processo de amamentação.

Por outro lado, o vínculo mãe-bebê, reforçado durante a prática da amamentação natural, pode se tornar tão intenso a ponto de dificultar o desmame, caracterizado nesses casos como um ato de separação, afastamento e abandono, e sendo, muitas vezes, mais doloroso emocionalmente para a mãe do que para a própria criança. Nos casos em que a amamentação natural ultrapassa os doze meses de idade, sendo considerada prolongada, além da maior dificuldade para o desmame, a extensão desse hábito pode trazer conseqüências negativas, como a recusa de alimentos sólidos por parte da criança (Brakohiapa, Yartiy, Bille, Harrison, Quansalh, Armar, Kishi \& Yamamoto, 1988), a subnutrição (Victora, Vaughan, Martines \& Barcelos, 1984) e a maior ocorrência de cáries (Li, Wang \& Caufield, 2000).

Apesar dos riscos, alguns pesquisadores, baseados em estudos com populações expostas a precárias condições socioeconômicas, consideram adequada a amamentação até os dois anos de vida da criança. O impacto positivo da amamentação prolongada sobre o processo de crescimento físico dessas crianças (Lima, Motta, Santos \& Silva, 2004) e o fato de o leite materno constituir a única fonte segura 
de alimento para as elas - considerando-se a existência de riscos significativos de contaminação durante a preparação de outros alimentos (Rao \& Kanade, 1992) - são alguns dos aspectos destacados pela literatura.

Segundo Caldeira e Goulart (2002), as variáveis que afetam ou influenciam o desmame precoce ou a extensão da amamentação podem ser divididas em cinco categorias: (a) variáveis demográficas: tipo de parto, idade materna, presença paterna na estrutura familiar, números de filhos, experiência com amamentação; (b) variáveis socioeconômicas: renda familiar, escolaridade materna e paterna, tipo de trabalho do chefe de família; (c) variáveis associadas à assistência pré-natal: orientação sobre amamentação, desejo de amamentar; (d) variáveis relacionadas à assistência pós-natal imediata: alojamento conjunto, auxílio de profissionais da saúde, dificuldade iniciais; e (e) variáveis relacionadas à assistência pós-natal tardia (após a alta hospitalar): estresse e ansiedade materna, uso de medicamentos pela mãe e pelo bebê, introdução precoce de alimentos.

A experiência clínica demonstra que enquanto uma parcela das mães desmama seus filhos precocemente, deixando de beneficiá-los de todas as vantagens da amamentação natural, outra parte realiza o aleitamento materno com tanto sucesso que muitas vezes apresenta uma certa dificuldade na realização do desmame, levando à extensão da amamentação natural. O objetivo deste estudo foi identificar fatores que influenciam a ocorrência do desmame precoce e da extensão da amamentação natural.

\section{Método}

Participaram deste estudo 80 mães de nível socioeconômico médio e baixo, residentes na cidade de Piracicaba, SP, e região, que acompanhavam seus filhos a um centro de pesquisa e atendimento odontológico para pacientes especiais. Dois grupos de 40 mães foram selecionados: (a) grupo I - composto por 40 mães que desmamaram seus filhos precocemente, isto é, antes do sexto mês de vida da criança; e (b) grupo II - composto por 40 mães que estavam realizando o aleitamento materno além do primeiro ano de vida da criança.

A amostra do grupo I foi selecionada a partir de 158 mães que ingressaram no Cepae no período de março de 2002 a outubro de 2003. Das 158 mães, 51 desmamaram seus filhos antes do sexto mês de vida e 40 (78\% da amostra elegível) foram selecionadas aleatoriamente. A amostra do grupo II foi selecionada a partir de 347 mães que participavam do Cepae nesse mesmo período e que tinham filhos com mais de um ano de idade. Das 347 mães, 43 estavam amamentando seus filhos além do primeiro ano de vida e 40 (representando 93\% da amostra elegível) foram selecionadas aleatoriamente.

As mães foram entrevistadas individualmente e em uma única sessão de atendimento. As entrevistas foram integralmente gravadas em fitas de áudio (K-7) para registro de maior número de dados e posterior transcrição ipsis literis. Inicialmente, foram obtidas informações a respeito do desmame precoce para as mães do primeiro grupo e sobre o aleitamento materno prolongado para as mães do segundo grupo, por meio de questionários específicos. Em seguida, foi aplicado um questionário socioeconômico (Kozlowski, 2001), que foi respondido por escrito e individualmente pelas mães a fim de garantir privacidade, evitando-se constrangimentos durante a escolha das respostas do questionário. O tratamento estatístico dos dados foi efetuado por meio dos Testes Qui-Quadrado e Exato de Fischer.

O projeto de pesquisa foi submetido à apreciação do Comitê de Ética em Pesquisa da Faculdade de Odontologia de Piracicaba, da Universidade Estadual de Campinas (Unicamp), tendo sido aprovado pelo protocolo nº14/2002.

\section{Resultados}

Quando as mães foram questionadas sobre seus sentimentos durante o ato de amamentar, antes da ocorrência do desmame, 76,0\% das mães pertencentes ao grupo de desmame precoce relataram que sentiam alegria, prazer ou realização pessoal, enquanto apenas 15,0\% referiram medo e/ou preocupação durante a amamentação, uma vez que acreditavam que o leite não estava sendo suficiente ou não sustentava o bebê. Segundo dados obtidos neste estudo, o desmame não foi planejado em $92,0 \%$ dos casos. Mais de $80,0 \%$ das mães pertencentes ao grupo de aleitamento materno prolongado referiram que sentiam alegria, prazer ou realização pessoal durante a amamentação de seus filhos (Figura 1). 
Ao comparar a pretensão da mãe, durante a gestação, em amamentar seu filho, não foi encontrada diferença estatística (Teste Exato de Fisher - $p>0,05$ ) entre os dois grupos estudados (95,0\% para desmame precoce e 92,5\% para aleitamento materno prolongado).

Também não foi encontrada diferença estatisticamente significante (Teste Qui-Quadrado - $p>0,05$ ) entre os dois grupos no que se refere ao recebimento de informação sobre amamentação durante a gestação, sugerindo que a disponibilidade de informação sobre amamentação não interferiu na sua interrupção ou extensão. Dois terços $(67,0 \%)$ das mães do grupo de desmame precoce e $57,5 \%$ das mães do grupo de aleitamento materno prolongado receberam informação sobre amamentação durante a gestação.

Neste estudo, o tipo de parto foi mais uma variável provavelmente não relacionada diretamente ao desmame precoce ou ao aleitamento materno prolongado, já que não foi detectada diferença estatística entre o tipo de parto nos dois grupos estudados ( $p=0,6802)$. Os números de cesáreas (55\%) e parto normal (45\%) foram idênticos em ambos.

Observou-se diferença estatística ( $p=0,0175)$ entre o número total de filhos das mães pertencentes aos dois grupos (Figura 2). Os dados apontaram que as mães que desmamaram seus filhos antes dos seis meses tinham menos filhos do que as mães que amamentaram além dos doze meses de vida do bebê.

Encontrou-se diferença estatisticamente significante $(p=0,0058)$ ao comparar o número de mães que já haviam amamentado previamente, pelo menos, um filho até o sexto mês de vida entre os dois grupos. No grupo de desmame precoce, havia um menor número de mães que realizaram a amamentação natural com sucesso (17,5\%), enquanto que no grupo de aleitamento materno prolongado, existia um maior número de mães com experiência prévia em aleitamento materno (45,0\%).

Os dados também mostraram uma diferença estatisticamente significante entre o estado civil das mães pertencentes aos dois grupos ( $p=0,0078$ ). Observa-se um maior percentual de mães solteiras no grupo de desmame precoce $(83,3 \%$ ) e uma tendência inversa, isto é, um maior percentual de mães casadas no grupo de aleitamento materno prolongado (56,7\%) (Figura 3).

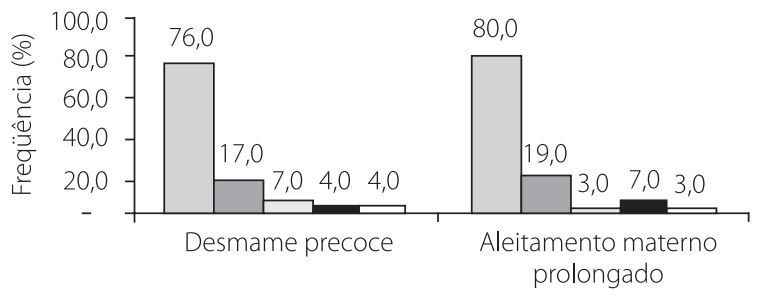

Duração da amamentação natural

\begin{tabular}{|ll|}
\hline$\square$ Alegria, prazer, realização pessoal & $\square$ Medo, preocupação \\
$\square$ Indiferença & $\square$ Raiva \\
$\square$ Tristeza & \\
\hline
\end{tabular}

Figura 1. Associação entre o sentimento vivenciado pela mãe durante o ato do aleitamento e a duração da amamentação (Teste Exato de Fischer $p>0,05$ ).

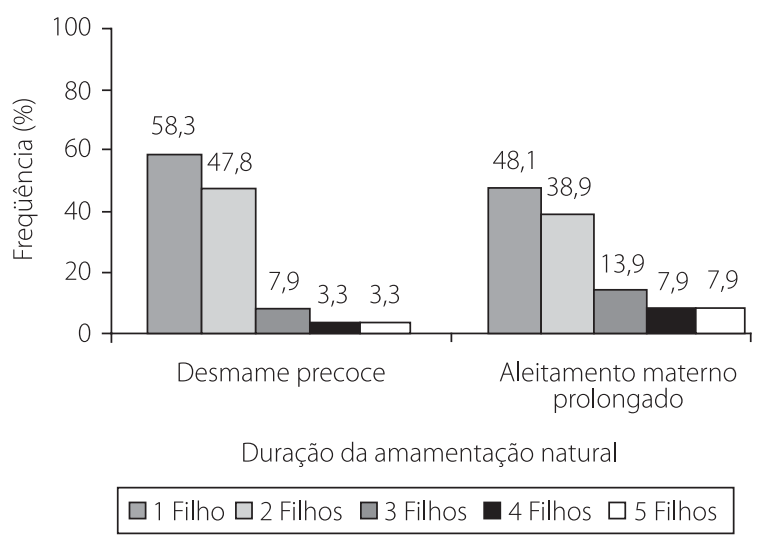

Figura 2. Associação entre o número total de filhos e a duração da amamentação (Teste Exato de Fischer $p<0,05$ ).

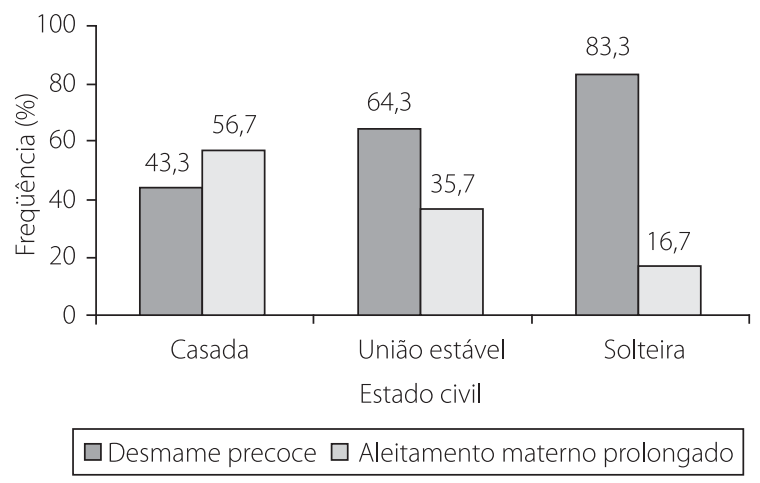

Figura 3. Duração da amamentação em função do estado civil da mãe (Teste Exato de Fischer $p<0,05$ ). 
Ao comparar a idade das mães (Figura 4), obteve-se uma diferença estatística significante $(p=0,0331)$. As mães pertencentes ao grupo de desmame precoce possuíam idade inferior (média de 22,6 anos) às mães do grupo de aleitamento materno prolongado (média de 27,2 anos).

Em relação à condição socioeconômica, verificou-se que a maioria das mães do grupo de desmame precoce pertencia à classe média, enquanto que a maior parte das mães do grupo de aleitamento materno prolongado pertencia à classe média inferior. Os dados sugerem que a condição socioeconômica está associada negativamente à duração da amamentação natural (Figura 5).

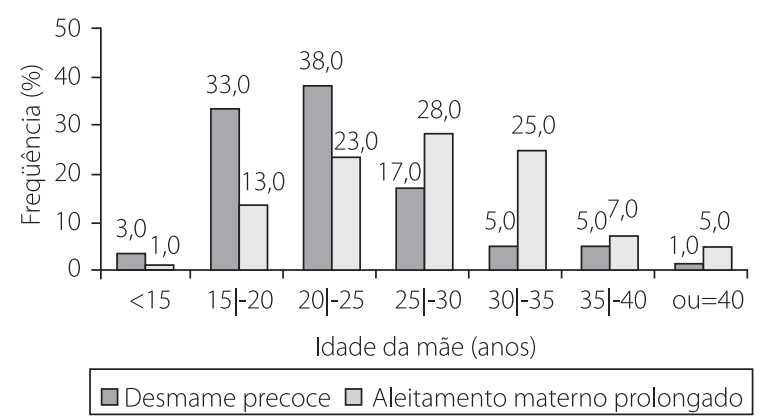

Figura 4. Associação entre a duração da amamentação e a idade da mãe quando do nascimento do filho (Teste Exato de Fischer $p<0,05$ )

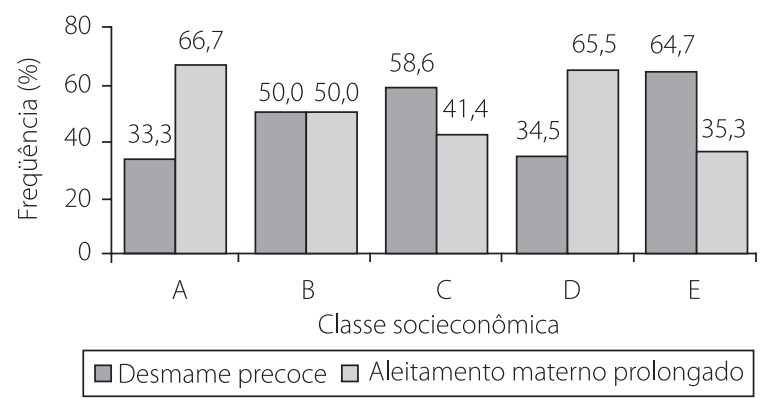

Figura 5. Associação entre a duração da amamentação e a condição socioeconômica dos pais (Teste Exato de Fischer $p<0,05)$

Nota: (A) Classe alta; (B) Classe média superior; (C) Classe média; (D) Classe média inferior; (E) Classe baixa.

\section{Discussão}

Os dados apontaram que a maioria das mães, de ambos os grupos, sentia prazer em amamentar e não planejou o desmame, não o fazendo por vontade própria, ao contrário dos resultados descritos por Ichisato e Shimo (2002), segundo os quais o desmame se refere a uma decisão tomada pela mulher, de forma consciente, embora essa conscientização seja negada.

Quando se comparou o sentimento das mães do grupo de desmame precoce com o do grupo de aleitamento materno prolongado, durante a amamentação, observou-se que, em ambos, a maior parte das mães referiu alegria, prazer ou realização pessoal. Esse dado sugere que o sentimento da mãe durante o ato da amamentação não parece ser um fator relacionado à extensão do aleitamento materno, pois as mães do grupo de desmame precoce, embora sentissem prazer em amamentar seus filhos, efetuaram o desmame. Novos estudos devem ser realizados para investigar a influência dessa variável na prática da amamentação natural, já que a literatura científica sobre o assunto é escassa.

O fato de não ter sido encontrada diferença estatística entre os dois grupos ao avaliar o desejo das mães, durante a gestação, de amamentarem seus filhos, pode ser interpretado de duas formas: primeiro, a intenção das mães de amamentar seu filho não constitui um fator que influencia o desmame precoce ou a extensão da amamentação natural; e, segundo, quando questionadas por profissionais de saúde, as mães (e respondentes de modo geral) tendem a apresentar respostas mais socialmente convenientes. Nesse caso, as mães poderiam até não ter intenção de amamentar, mas como existe um conceito divulgado e valorizado pela sociedade de que a mulher deve amamentar seu filho, elas se percebem na obrigação de responder conforme essa linha de pensamento, isto é, compatível com a expectativa social estabelecida. Assim, pode-se sugerir que algumas das mães que relataram ter a intenção de amamentar seus filhos não foram sinceras, apenas oferecendo a resposta que consideravam, socialmente, a mais adequada (Faleiros, Kalil, Casarin, Laque-Júnior \& Santos, 2005).

A literatura aponta divergências em relação à influência do pré-natal na duração da amamentação 
natural. O trabalho de Granzoto, Bertoni, Vecchi e Rodrigues (1992) identificou o pré-natal como uma variável eficiente na prevenção da ocorrência do desmame precoce, enquanto os trabalhos de Gomes, Cardoso, Moura e Marçal (1992) e Giugliane, Rocha, Neves, Palanczyk, Seffin e Susin (1995) apontaram que o grau de conhecimento adquirido pela mãe durante a gestação não está relacionado ao sucesso da amamentação e à duração do aleitamento materno.

Analisando os três trabalhos referidos no parágrafo anterior, é possível sugerir que a orientação, durante o pré-natal, seja relevante para os seguintes aspectos: (a) familiarização das gestantes quanto ao papel do aleitamento materno para sua própria saúde e a do bebê; (b) preparação da mama para o ato de amamentar; (c) necessidade de permanência em alojamento conjunto após o parto; e (d) efeitos deletérios do uso de mamadeira, chupeta e outros hábitos orais. Todavia, é extremamente necessária a atuação de grupos de incentivo ao aleitamento materno a fim de reforçar o conteúdo explicitado durante o pré-natal, de disponibilizar apoio psicossocial às mães e de solucionar os inúmeros problemas que surgem durante os primeiros dias e meses após o parto. $\mathrm{O}$ incentivo realizado durante o pré-natal torna-se potencialmente mais útil quando seguido de um acompanhamento periódico e sistematizado após o nascimento do bebê.

A ausência de associação entre o tipo de parto e a duração da amamentação, verificada neste estudo, está de acordo com o trabalho de Weiderpass, Barros, Victora, Tomasi e Halpern (1998), que também não encontrou diferença na prevalência de amamentação natural conforme o tipo de parto. Os dados do trabalho de Rowe-Murray e Fisher (2002) contrastam com os resultados desta pesquisa e do autor acima citado. Os autores observaram que a cesariana foi um fator de risco para o início da lactação, pois esse tipo de parto implicou o aumento do uso de anestésicos e analgésicos que retardaram o primeiro contato mãe-filho e o estabelecimento da amamentação. Além disso, acarretou uma recuperação mais difícil, gerando maior desconforto físico da mãe ao lidar com o bebê.

Segundo DiGirolamo, Grummer-Strawn e Fein (2001), variações nas práticas hospitalares podem explicar essas diferenças. Hospitais cujo padrão de atendimento pós-operatório dificulta o alojamento conjunto e o aleitamento à livre-demanda, ou, ainda, permite a introdução precoce de outros alimentos na dieta infantil, por exemplo, podem levar a uma maior probabilidade de insucesso do aleitamento.

Os dados desta pesquisa sugerem que mães que já amamentaram, pelo menos, um outro filho com sucesso têm maiores chances de estender a amamentação, enquanto aquelas que nunca tiveram tal experiência têm maior probabilidade de realizar o desmame precocemente. Confirmando esse resultado, Barros, Semer, Tonieli e Victora (1995) identificaram uma maior freqüência de amamentação em mães que possuíam mais de um filho. Lawoyin, Olawuyi e Onadeko (2001), bem como Venâncio, Escuder, Kitoko, Rea e Monteiro (2002), verificaram que mulheres primíparas tinham maiores chances de abandonar o aleitamento materno antes de a criança completar quatro meses de vida e estavam mais propensas a introduzir outro tipo de alimento na dieta de seus filhos antes do sexto mês de vida. Meyerink e Marques (2002) destacaram que o início e o sucesso da amamentação natural estavam fortemente relacionados ao fato de a mãe ter amamentado, pelo menos, uma criança previamente. Segundo esses autores, a chance de sucesso no aleitamento materno foi dez vezes maior entre as mães com experiência, quando comparadas àquelas que não amamentaram um filho anteriormente.

Por outro lado, Ekström, Widström e Nissen (2003) não encontraram diferença na extensão da amamentação quando compararam um grupo de primíparas com outro de multíparas até que as crianças completassem três meses de vida. O estudo de Arantes (1995) destacou que a mulher não pode ser rotulada de experiente ou inexperiente pelo fato de ter ou não vivenciado a amamentação, pois cada vivência inclui aspectos subjetivos que são únicos a cada filho amamentado. Para Arantes, na abordagem do profissional de saúde com a mulher, independente do número de filhos, é necessário incentivá-la a refletir sobre a vivência da amamentação nos diferentes momentos de sua existência.

Resultados deste estudo também permitem sugerir que quanto maior a estabilidade conjugal, maior a chance de a mãe estender a amamentação natural, diminuindo os riscos da ocorrência de desmame precoce. De modo semelhante, Zimmermam e Guttman (2001) destacaram que o sucesso nas práticas de aleitamento materno estava relacionado à estabilidade 
conjugal dos pais, isto é, mães casadas tinham maiores chances de iniciar e estender a amamentação natural. Cernadas et al. (2003) apontaram que o suporte familiar constituía um aspecto extremamente relevante na prática do aleitamento natural, sendo que o principal envolvido era o companheiro. Os autores concluíram que mães que eram encorajadas e recebiam um adequado apoio familiar apresentavam maiores chances de realizar a amamentação natural com sucesso, pelo menos, até o sexto mês de vida da criança.

A literatura é relativamente contraditória ao associar a idade materna com a duração da amamentação natural. Os trabalhos de Gigante, Victora e Barros (2000), Lawoyin, Olawuyi e Onadeko (2001) e Zimmermam e Guttman (2001) confirmam os dados obtidos neste estudo: a prevalência de amamentação natural é maior com o aumento da idade materna. Já os trabalhos de Venâncio et al. (2002), bem como de Cernadas, Noceda, Barreira, Martinez e Garsd (2003), não encontraram relação entre aleitamento materno e idade da mãe.

Ao comparar a condição socioeconômica entre os dois grupos deste estudo, observou-se que a maioria das famílias do grupo de desmame precoce pertencia à classe média, enquanto a maioria das famílias do grupo de aleitamento materno prolongado pertencia à classe média inferior. Essa diferença estatisticamente significante se deve, provavelmente, ao número reduzido de famílias das classes sociais A e B. Este estudo utilizou uma amostra tipificada, constituída de participantes que eram atendidos por um serviço público de saúde que abrange, principalmente, integrantes das classes sociais C, De E.

Os trabalhos de Kummer, Giugliane, Susin, Folletto, Lermen e Wu (2000) e de Li et al. (2000) identificaram uma menor duração da amamentação em populações de baixa condição socioeconômica, confirmando os dados do presente estudo. Por outro lado, o trabalho de Gigante, Victora e Barros (2000) não identificou relação entre a duração do aleitamento materno e a condição socioeconômica.

\section{Considerações Finais}

Os dados obtidos por este estudo permitiram a elaboração das seguintes considerações finais: (a) informações sobre amamentação durante o pré-natal, desejo de amamentar, tipo de parto e os sentimentos vivenciados pela mãe durante o ato da amamentação natural não eram fatores diretamente relacionados à extensão da amamentação natural; (b) fatores como estado civil dos pais, idade materna, número total de filhos e experiência em amamentação (ter amamentado, pelo menos, um filho até seis meses de vida) eram fatores que predispõem à ocorrência do desmame precoce ou da extensão do aleitamento materno. Dessa forma, variáveis demográficas podem constituir preditores relevantes do sucesso ou insucesso da amamentação natural. Destaca-se, ainda, que tais variáveis podem ser identificadas durante o período pré-natal de modo a priorizar as gestantes com alto risco para a ocorrência do desmame precoce.

\section{Referências}

Arantes, C.I.S. (1995). Amamentação: visão das mulheres que amamentam. Jornal de Pediatria, 71, 195-202.

Araújo, M.F.M., Fiaco, A.D., Pimentel, L.S., \& Schmitz, B.A.S. (2004). Custo e economia da prática do aleitamento materno para a família. Revista Brasileira de Saúde Materno Infantil, 4 (2), 135-141.

Barros, F.C., Semer, T.C., Tonieli, S., Tomasi, E., \& Victora, C.G. (1995). The impact of lactation centres on breastfeeding patterns, morbidity and growth: A birth cohort study. Acta Paediatric, 84 (11), 1221-1226.

Brakohiapa, B., Yartey, J., Bille, A., Harrison, E., Quansah, E., Armar, M.A., Kishi, K., \& Yamamoto, S. (1988). Does prolonged breastfeeding adversely affect a child's nutritional status? Lancet North American, 20 (2), 416-418.

Caldeira, A.P., \& Goulart, E.M.A. (2000). A situação do aleitamento materno em Montes Claros, Minas Gerais: estudo de uma amostra representativa. Jornal de Pediatria, $76(1), 65-72$

Camilo, D.F., Carvalho, RV.B., Oliveira, E.F., \& Moura, E.C. (2004). Prevalência da amamentação em crianças menores de dois anos vacinadas nos centros de saúde escola. Revista de Nutrição, 17 (1), 29-36.

Cernadas, J.M., Noceda, G., Barreira, L., Martinez, A.M., \& Garsd, A. (2003). Maternal and perinatal factors influencing the duration of exclusive breastfeeding during the first 6 months of life. Journal of Human Lactation, 19 (2), $136-44$.

Dennis, C.L. (2002). Breastfeeding peer support: maternal and volunteer perceptions for a randomized controlled trial. Birth, 29 (3), 169-176.

DiGirolamo, A.M., Grummer-Strawn, L.M., \& Fein, S. (2001). Maternity care practices: implications for breastfeedings. Birth, 28 (2), 94-100. 
Ekström, A., Widström, A.M., \& Nissen, E. (2003). Duration of breastfeeding in swedish primiparous and multiparous women. Journal of Human Lactation, 19 (2), 172-178.

Faleiros, J.J., Kalii, G., Casarin, D.P., Laque-Júnior, P.A., \& Santos, I.S. (2005). Avaliação do impacto de um programa de puericultura na promoção da amamentação exclusiva. Cadernos de Saúde Pública, 21 (2),482-489.

Gigante, D.P., Victora, C.G., \& Barros, F.C. (2000). Nutrição materna e duração da amamentação em uma coorte de nascimentos de Pelotas-RS. Revista de Saúde Pública, 34 (3), 259-265

Giugliane, E.R.J., \& Victora, C.G. (2000). Alimentação complementar. Jornal de Pediatria, 76 (Suppl 3), 253-262.

Giugliane, E.R.J., Rocha, V.L.L., Neves, J.M., Polanczyk, C.A., Seffrin, C.F., \& Susin, L.O. (1995). Conhecimentos maternos em amamentação e fatores associados. Jornal de Pediatria, 71 (2), 77-81.

Gomes, A.C.S., Cardoso, M.L., Moura, E.F.A., \& Marçal, N.K. (1992). Aleitamento ao seio. Jornal de Pediatria, 68 (3/4), 123-126.

Granzoto, J.A., Bertoni, A.L., Vecchi, A.A., \& Rodrigues, E. (1992). A importância do incentivo pré-natal na amamentação de primípras. Jornal de Pediatria, 68 (1/2), 34-37.

Ichisato, S.M.T., \& Shimo, A.K.K. (2002). Revisitando o desmame precoce através de recortes da história. Revista Latino Americana de Enfermagem, 10 (4), 578-585.

Kozlowski F.C. (2001). Relação entre o fator socioeconômico e a prevalência e a severidade de fluorose e cárie dentária. Dissertação de mestrado não-publicada, Universidade Estadual de Campinas, Piracicaba.

Kummer, S.C., Giugliane, E.R.J., Susin, L.O., Folletto, J.L., Lermen, N.R., \&Wu, V.Y.J. (2000). Evolução do padrão de aleitamento materno. Revista de Saúde Pública, 34 (2), 143-148.

Lawoyin, T.O., Olawuyi, J.F., \& Onadeko, M.O. (2001). Factors associated with exclusive breastfeeding in Ibadan, Nigéria. Journal of Human Lactation, 17 (4), 321-325.

Leite, A.M., Silva, I.A., \& Scochi, C.G.S. (2004). Comunicação não-verbal: uma contribuição para o aconselhamento em amamentação. Revista Latino-Americana de Enfermagem, 12 (2), 258-264.

Li, Y., Wang, W., \& Caufield, P.W. (2000). The fidelity of mutans streptococci transmission and caries status correlate with breast-feeding experience among chinese families. Caries Research, 34 (2), 123-132.

Lima, M.C., Motta, M.E.F.A., Santos, E.C., \& Silva, G.A.P. (2004). Determinants of impaired growth among hospitalized children: a case-control study. São Paulo Medical Journal, $122(3), 117-123$.
Meyerink, R.O., \& Marquis, G.S. (2002). Breastfeeding initiation and duration among low-income women in Alabama: the importance of personal and familial experiences in making infant-feeding choices. Journal of Human Lactation, 18 (1), 38-44.

McKeever, P., Stevens, B., Miller, K.L., McDonell, J.W., Gibbins, S., Guerriere, D., Dunn, M.S., \& Coyte, P.C. (2002). Home versus hospital breastfeeding support for newborns: a randomized controlled trial. Birth, 29 (4), 258-265.

Nascimento, M.B.R., \& Issler, H. (2003). Breastfeeding: making the difference in the development, health and nutrition of term and preterm newborns. Revista do Hospital das Clínicas da Faculdade de Medicina de São Paulo, 58 (1), 49-60.

Neighbors, K.A., Gillespie, B., Schwartz, K., \& Foxman, B. (2003). Weaning practices among breastfeeding women who weaned prior to six months postpartum. Journal of Human Lactation, 19 (4), 374-380.

Pedroso, G.C., Puccini, R.F., Silva, E.M.K., Silva, N.N., Alves, M.C.G.P. (2004). Prevalência de aleitamento materno e introdução precoce de suplementos alimentares em área urbana do sudeste do Brasil, Embu, SP. Revista Brasileira de Saúde Materno Infantil, 4(1), 45-58.

Rao, S., \& Kanade, A.N. (1992). Prolonged breast-feeding and malnutrition among rural Indian children below 3 years of age. European Journal of Clinical Nutrtition, 46 (3), 187-195.

Rezende, M.A. (2004). Respirador bucal: uma visão clínica e funcional da amamentação. Revista Latino-Americana de Enfermagem, 12 (1), 139-141.

Rowe-Murray, H.J., \& Fisher, J.R.W. (2002). Baby friendly hospital practices: cesarean section is a persistent barrier to early initiation of breastfeeding. Birth, 29 (2), 124-131.

Updegrove, K. (2004). Necrotizing enterocolitis: the evidence for use of human milk in prevention and treatment. Journal of Human Lactation, 20 (3), 335-339.

Venâncio, S.I., Escuder, M.M.L., Kitoko, P., Rea, M.F., \&Monteiro, C. A. (2002). Freqüência e determinantes do aleitamento materno em municípios do Estado de São Paulo. Revista de Saúde Pública, 36 (3), 313-318.

Weiderpass, E., Barros, F.C., Victora, C.G., Tomasi, E., \& Halpern, R. (1998). Incidência e duração da amamentação conforme o tipo de parto: estudo longitudinal no Sul do Brasil. Revista de Saúde Pública, 32 (3), 255-231.

Zimmerman, D.R., \& Guttman, N. (2001). "Breast is best": Knowledge among low-income mothers is not enough. Journal of Human Lactation, 17 (1), 14-19

Recebido para publicação em 17 de dezembro de 2004 e aceito em 2 de junho de 2005 